\title{
新型水溶性圆柱形大三环主体与联吡啶盐的络合性能研究
}

\author{
曾飞*, 唐琳俐 ${ }^{a}$ 陈小明 ${ }^{a}$ 李治章 ${ }^{a}$ 韩 莹 ${ }^{b}$ 陈传峰*,b \\ ( ${ }^{a}$ 湖南科技学院化学与生物工程学院 湘南优势植物资源综合利用湖南省重点实验室 永州 425199) \\ ( ${ }^{b}$ 中国科学院化学研究所 分子识别与功能院重点实验室 北京 100190)
}

\begin{abstract}
摘要 描述了新型水溶性圆柱形大三环主体与联吡啶盐客体在水溶液中与固体下的络合性质, 表明大三环主体与联吡 啶盐能够形成 $1: 1$ 的超分子络合物. 此外, 主客体络合物的形成与解离还可以通过酸碱的加入进行可逆调控, 并且该 过程也可通过肉眼进行观察.
\end{abstract}

关键词 大三环主体; 联吡啶盐; 络合作用; 酸碱调控

\section{Complexation of Novel Water-Soluble Cylindrical Macrotricyclic Host and Paraquat}

\author{
Zeng, Fei ${ }^{*, a} \quad{\text { Tang, } \text { Linli }^{a} \quad \text { Chen, Xiaoming }}^{a} \quad$ Li, Zhizhang ${ }^{a}$ \\ Han, Ying ${ }^{b} \quad$ Chen, Chuanfeng ${ }^{*, b}$
}

$\left({ }^{a}\right.$ Key Laboratory Comprehensive Utilization of Dominant Plants Resources in South Hunan, Department of Biology and

Chemistry, Hunan University of Science and Engineering, Yongzhou 425199)

( ${ }^{b}$ CAS Key Laboratory of Molecular Recognition and Function, Institute of Chemistry, Chinese Academy of Sciences, Beijing 100190)

\begin{abstract}
The complexation between the novel water-soluble cylindrical macrotricyclic host and paraquat guests in both solution and solid state was investigated in detail. It was found that the host could form $1: 1$ complexes with paraquat salts in water solution and solid state. Interestingly, the complexation and decomplexation of the complexes between the host and the guest could be achieved by changing the $\mathrm{pH}$ of the solution, and the process could also be observed by naked eye.
\end{abstract}

Keywords macrotricyclic host; paraquat; complexation; acid-base controlled

1967 年, Pedersen ${ }^{[1]}$ 首次合成了冠醚并发现其能识 别金属离子, 标志着大环化学的诞生, 拉开了主客体化 学研究的序幕, 是超分子化学纪元的开始. 发展基于大 环主体分子的新型超分子体系始终是超分子化学发展 的推动力和重要内容, 探索合成新的大环主体分子并研 究其功能始终是超分子化学家的首要任务 ${ }^{[2]}$. 开展在水 溶液中的主客体化学研究, 对了解生物体内的代谢过程 具有更重要的现实意义, 引起了超分子化学家们广泛的 研究兴趣. 目前, 用于水溶液中主客体化学研究的大环 主体分子主要是水溶性的环糊精 ${ }^{[3]}$ 和葫芦脲 ${ }^{[4]}$ 以及修饰 的杯芳烃 ${ }^{[5]}$ 和柱芳烃 $^{[6]}$ 等. 冠醚作为超分子化学中的第 一代主体分子, 在 40 多年的研究中基于冠醚在有机溶
剂中的识别性能及超分子组装体取得了丰硕的成果 ${ }^{[7]}$, 而在水溶液中的主客体化学研究相对较少 ${ }^{[8]}$. 目前, 基 于冠醚在水溶液中的主客体化学研究主要是单环冠醚 对联吡啶盐衍生物的识别作用, 且络合常数相对较少 (小于 $10^{3} \mathrm{~L} \cdot \mathrm{mol}^{-1}$ ).

在前面的工作中, 我们合成了带羧酸基团的四臂大 三环冠醚主体 $\mathbf{1}^{[9]}$, 并研究了主体分子 1 与喹啉盐以及 异喹啉盐之间的络合性质, 发现主体分子 $\mathbf{1}$ 能够与客体 分子在水溶液中与固态下形成 $1: 2$ 的络合物. 百草枯 及其衍生物(联吡啶盐衍生物)是一种快速灭生性除草 剂, 对人体毒性极大, 且无特效解毒药. 因此, 在本工 作中, 我们将研究大三环主体 $\mathbf{1}$ 与联吡啶盐衍生物在水

*E-mail: zengfei@iccas.ac.cn; cchen@iccas.ac.cn

Received March 15, 2016; revised March 30, 2016; published online April 15, 2016.

Project supported by the Sci-Tech Innovation Teams in Universities of Hunan Province and the Construct Program of the Key Discipline in Hunan Province (2016).

湖南省高校科技创新团队及湖南省有机化学重点学科(2016)资助项目. 
溶液中与固态下的络合作用, 发现大三环主体分子与联 吡啶盐衍生物在水溶液中与固态下能形成稳定的 $1: 1$ 超分子络合物, 络合常数较大 (大于 $10^{3} \mathrm{~L} \cdot \mathrm{mol}^{-1}$ ), 且络 合物的形成与解离可以通过酸碱的加入进行可逆调控.
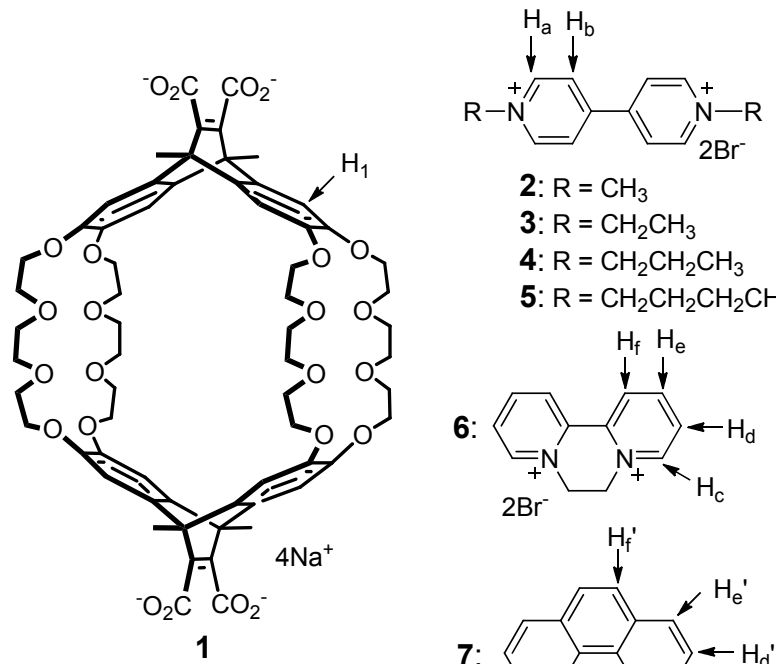

2: $\mathrm{R}=\mathrm{CH}_{3}$

3: $\mathrm{R}=\mathrm{CH}_{2} \mathrm{CH}_{3}$

4: $\mathrm{R}=\mathrm{CH}_{2} \mathrm{CH}_{2} \mathrm{CH}_{3}$

5: $\mathrm{R}=\mathrm{CH}_{2} \mathrm{CH}_{2} \mathrm{CH}_{2} \mathrm{CH}_{3}$

6:
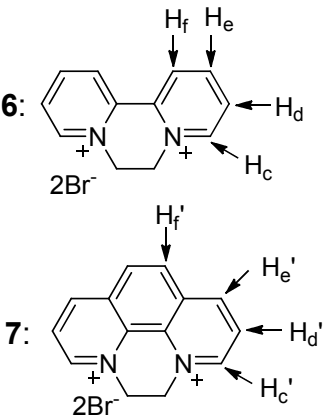

图 1 大三环主体 1 与客体 $\mathbf{2} \sim 7$ 的结构以及质子信号标注

Figure 1 Chemical structures and proton designations of host 1 and guests $\mathbf{2} \sim 7$

\section{1 结果与讨论}

\section{1 主体 1 与联吡啶盐在溶液中络合性质的研究}

首先, 我们研究了主体分子 $\mathbf{1}$ 与客体 $\mathbf{2}$ 在溶液中的 识别作用. 室温下, 当把主体 1 与客体 2 按 1：1 物质的 量之比混合在水溶液中, 发现溶液的颜色由原来的浅黄 色变为棕黄色, 这表明富电子主体与缺电子客体之间发 生了电荷转移相互作用. 随后, 我们用 ${ }^{1} \mathrm{H}$ NMR 考察了 主体分子 1 与客体 2 在溶液中的络合行为. 如图 2 所示, ${ }^{1} \mathrm{H}$ NMR 结果表明, 当在室温条件下, 主体 $\mathbf{1}$ 与客体 $\mathbf{2}$ 以 $1: 1$ 物质的量之溶于気代水中, 其 ${ }^{1} \mathrm{H}$ NMR 显示出 一组不同于游离主体与游离客体的质子信号, 这表明主 体分子和客体分子形成了一个新的络合物 $\mathbf{1 \cdot 2}$, 而且该 络合过程是一个快速过程. 主体上质子 $\mathrm{H}_{1}$ 的核磁信号 明显地向高场发生了位移 $(\Delta \delta=-0.34)$, 这可能是由于 主体分子中富电子的芳环与缺电子的联吡啶盐片段产 生的 $\pi-\pi$ 堆积作用导致的. 同时, 联吡啶盐上的质子 $\mathrm{H}_{\mathrm{a}}$ 和 $\mathrm{H}_{\mathrm{b}}$ 的核磁信号也明显向高场移动, 说明客体分子处 于主体分子空腔的屏蔽区. 这些实验结果都表明主客体 之间形成了稳定的络合物. 为了得到主体分子与客体分 子之间络合的定量关系, 我们利用摩尔比方法, 对主体 1 与客体 2 在気代水溶液中的络合行为进行了核磁滴 定实验, 发现主体 1 与客体 2 的络合比为 1 : 1. 进一步
利用 Scatchard 方程, 以 $P$ 为横坐标，以 $P /[2]$ 值为纵坐 标作图, 求得 $1 \cdot 2$ 的络合常数 $K_{\mathrm{a}, \exp }$ 为 $(1.1 \pm 0.07) \times 10^{4}$ $\mathrm{mol}^{-1} \cdot \mathrm{L}$.

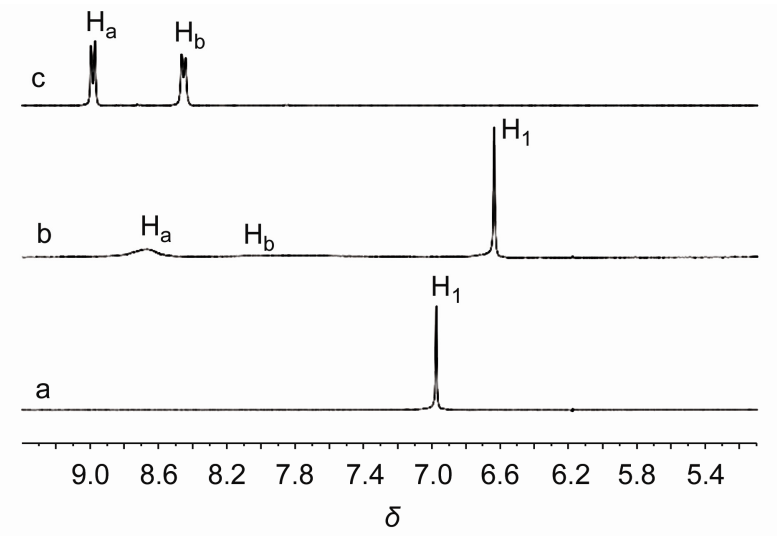

图 2 室温下(a)主体 1、(b)主体 1 和 1.0 equiv.的客体 2、(c) 客体 2 在 $\mathrm{D}_{2} \mathrm{O}$ 中的部分氢谱图 $(300 \mathrm{MHz})\left([1]_{0}=4.0 \mathrm{mmol} / \mathrm{L}\right)$ Figure 2 Partial ${ }^{1} \mathrm{H}$ NMR spectra $(300 \mathrm{MHz}, 298 \mathrm{~K})$ in $\mathrm{D}_{2} \mathrm{O}$ of (a) free $\mathbf{1}$, (b) $\mathbf{1}$ and 1.0 equiv. of $\mathbf{2}$, and (c) free 2 ([1 $]_{0}=4.0$ $\mathrm{mmol} / \mathrm{L}$ )

类似地，我们还通过相同的方法研究了主体 1 与其 他联吡啶盐客体在溶液中的络合作用. 结果表明, 客体 3 5 可以与主体形成稳定的 1:1 超分子络合物. 通过 Scatchard 方程 ${ }^{[10]}$, 计算出所有主客体络合物的络合常 数, 并总结在表 1 中.

表 1 络合物的络合比以及络合常数

Table 1 Summary of the stoichiometries and association constants of the complexes

\begin{tabular}{ccc}
\hline Complex & Stoichiometry $(\mathrm{H}: \mathrm{G})$ & $K_{\mathrm{a}} /\left(\mathrm{mol}^{-1} \cdot \mathrm{L}\right)$ \\
\hline $\mathbf{1 \bullet 2}$ & $1: 1$ & $(1.1 \pm 0.07) \times 10^{4}$ \\
$\mathbf{1 \bullet 3}$ & $1: 1$ & $(1.7 \pm 0.14) \times 10^{3}$ \\
$\mathbf{1 \bullet 4}$ & $1: 1$ & $(2.5 \pm 0.12) \times 10^{3}$ \\
$\mathbf{1 \bullet 5}$ & $1: 1$ & $(3.7 \pm 0.20) \times 10^{3}$ \\
$\mathbf{1 \bullet 6}$ & $1: 1$ & $(1.2 \pm 0.02) \times 10^{4}$ \\
$\mathbf{1 \bullet 7}$ & $1: 1$ & $(3.5 \pm 0.06) \times 10^{3}$ \\
\hline
\end{tabular}

此外, 我们还研究了主体分子 1 与另一种除草剂 1,1'-乙撑-2,2'-联吡啶盐 6 及客体分子 7 在溶液中的络合 作用. 在室温条件下, 当主体分子 1 与客体分子 $\mathbf{6}$ 在水 溶液中等物质的量混合后，溶液颜色由原来的浅黄色变 为棕红色, 这表明富电子主体与缺电子客体之间发生了 电荷转移相互作用; 同样, 主体 $\mathbf{1}$ 与客体 7 的混合溶液 也观察到了颜色的变化. 核磁氢谱对照实验研究结果显 示，当在室温条件下，主体分子 1 与客体分子 6 等物质 的量溶于氝代水中, 其核磁信号显示与主体分子和客体 分子都有很大的不同(如图 3), 主体上 $\mathrm{H}_{1}$ 和客体上的 $\mathrm{H}_{\mathrm{e}}$ 和 $\mathrm{H}_{\mathrm{f}}$ 明显地向高场移动, 说明了主客体之间存在比较 强的 $\pi-\pi$ 相互作用，客体分子处于主体分子空腔的屏蔽 区. 这些信息都表明主客体之间形成了稳定的 $1 \bullet \mathbf{6}$ 超分 
子络合物. 此外, 在主客体络合物的氢谱谱图上只有一 组络合物的核磁信号, 而并不能发现独立存在的主体分 子或者客体分子的信号, 这说明主客体的络合与解络合 是一个非常快速的过程. 利用摩尔比(mole ratio plot)方 法, 对主体 1 与客体 6 在氞代水溶液中的络合行为进行 了核磁滴定实验，发现主体 1 与客体 $\mathbf{6}$ 的络合比为 1 :

1. 进一步利用 Scatchard 方程, 以 $P$ 为横坐标, 以 $P /[\mathbf{6}]$ 值为纵坐标作图, 求得 $\mathbf{1 \cdot 6}$ 的络合常数 $K_{\mathrm{a}, \mathrm{exp}}$ 为(1.2 \pm $0.02) \times 10^{4} \mathrm{~mol}^{-1} \cdot \mathrm{L}$. 通过同样的实验, 发现主体 $\mathbf{1}$ 与客 体 7 的络合比为 $1: 1$, 络合常数 $K_{\mathrm{a}, \mathrm{exp}}$ 为 $(3.5 \pm 0.06) \times$ $10^{3} \mathrm{~mol}^{-1} \cdot \mathrm{L}$.

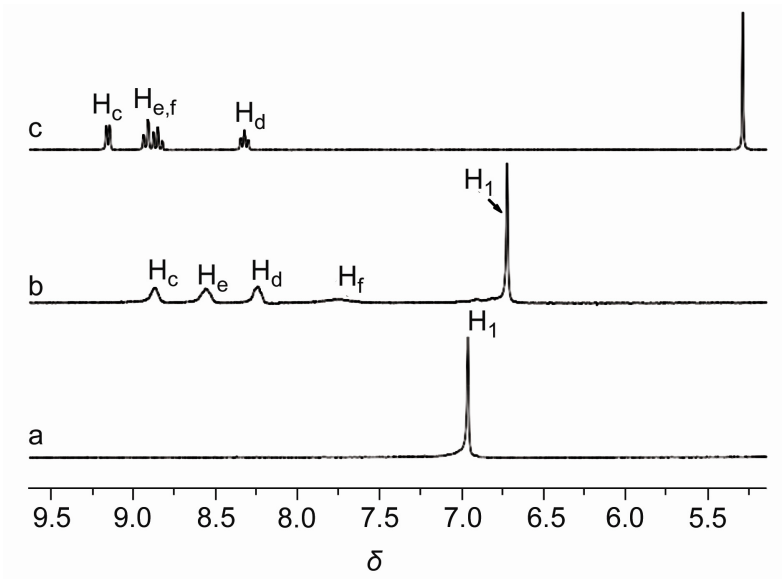

图 3 室温下 (a)主体 1、(b)主体 1 和 1.0 equiv. 的客体 $\mathbf{6}$ 及(c) 客体 6 在 $\mathrm{D}_{2} \mathrm{O}$ 中的部分氢谱图 $(300 \mathrm{MHz})\left([1]_{0}=4.0 \mathrm{mmol} / \mathrm{L}\right)$ Figure 3 Partial ${ }^{1} \mathrm{H}$ NMR spectra $(300 \mathrm{MHz}, 298 \mathrm{~K})$ in $\mathrm{D}_{2} \mathrm{O}$ of (a) free 1 , (b) 1 and 1.0 equiv. of 6 , and (c) free $6\left([1]_{0}=4.0\right.$ $\mathrm{mmol} / \mathrm{L}$ )

\section{2 主体 1 与甲基联吡啶盐 2 在固态下络合性质的研} 究

通过向主一客体的水溶液中扩散丙酮, 得到了可用 于 $\mathrm{X}$ 射线衍射实验的褐色的块状晶体单晶. 如图 5 所示,
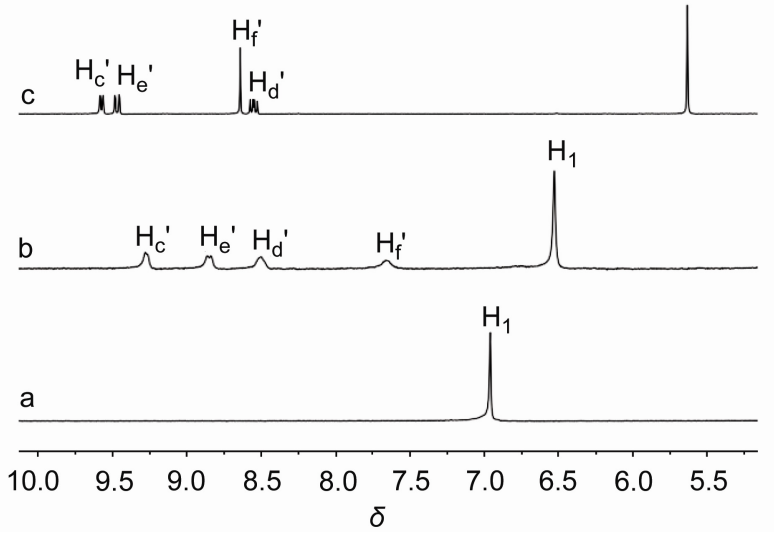

图 4 室温下(a)主体 1、(b)主体 1 和 1.0 equiv. 的客体 7 及(c) 客体 7 在 $\mathrm{D}_{2} \mathrm{O}$ 中的部分氢谱图 $(300 \mathrm{MHz})\left([1]_{0}=4.0 \mathrm{mmol} / \mathrm{L}\right)$ Figure 4 Partial ${ }^{1} \mathrm{H}$ NMR spectra $(300 \mathrm{MHz}, 298 \mathrm{~K})$ in $\mathrm{D}_{2} \mathrm{O}$ of (a) free $\mathbf{1}$, (b) $\mathbf{1}$ and 1.0 equiv. of 7 , and (c) free 7 ([1 $]_{0}=4.0$ $\mathrm{mmol} / \mathrm{L}$ )

客体分子甲基联吡啶盐刚好位于大环主体 1 的中心空 腔, 而且客体上的两个 $\mathrm{N}$ 甲基也刚好位于冠醚-24-冠-8 的中心位置, 络合物的结构类似于准轮烷结构. 另外, 我们发现在络合物中客体的两个芳环发生了扭曲不在 同一个平面上, 扭曲角度为 $9.10^{\circ}$. 主体分子与客体分子 芳环之间存在面对面的 $\pi \cdots \pi$ 堆积相互作用, $\mathrm{A}$ 到 $\mathrm{C}$ 以及 $\mathrm{B}$ 到 $\mathrm{D}$ 环的中心距离 $(\AA)$ 和二面角 $\left(^{\circ}\right)$ 分别为: $4.13(\mathrm{e})$, 26.53 和 4.12 (f), 31.38. 由于络合物中存在多重弱相互 作用, 使主客体络合物 $\mathbf{1 \cdot 2}$ 非常稳定, 与溶液中的稳定 状态相吻合.

\section{3 主体 1 对甲基联吡啶盐的络合与解离}

由于阴离子羧酸盐与中性翔基之间的转换可以通 过酸碱的加入来调节, 因此络合物 $1 \cdot 2$ 的络合与解离可 通过 $\mathrm{pH}$ 进行调控. 如图 6 所示, 向主客体络合物 $\mathbf{1 \cdot 2}$ 中 加入 $2.0 \mu \mathrm{L}$ 的気代盐酸后, 络合物的信号消失, 客体分 子 2 中 $\mathrm{H}_{\mathrm{a}}, \mathrm{H}_{\mathrm{b}}$ 氢质子信号向低场发生位移, 恢复到未络


图 5 络合物 $\mathbf{1 . 2}$ 的单晶结构(a)及其(b)俯视图、(c)侧视图

Figure 5 Crystal structure of complex $\mathbf{1 \cdot 2}$ (a), (b) top view and (c) side view Solvent molecules, $\mathrm{Na}^{+}$counterions, $\mathrm{Br}^{-}$and hydrogen atoms were omitted for clarity 
合时的值. 同时, 主体分子中 $\mathrm{H}_{1}$ 氢质子信号消失. 这些 结果表明, 络合物 1.2 发生了解离, 并生成了不溶于水 的质子化的主体分子. 当向上述体系中再加入 $1.0 \mu \mathrm{L}$ 的 质量分数为 $40 \%$ 的氛代氢氧化钠溶液后, 客体分子 2 中 $\mathrm{H}_{\mathrm{a}}, \mathrm{H}_{\mathrm{b}}$ 氢质子信号向高场位移, 恢复到络合时的值. 同 时, 主体分子发生去质子化的过程伴随着 $\mathrm{H}_{1}$ 氢质子信 号的出现, 重新形成络合物 $\mathbf{1 \bullet 2}$. 这种主体分子对客体 分子可逆的络合与解离还可以通过肉眼进行观察. 如图 6 所示, 向主客体络合物 $\mathbf{1 \cdot 2}$ 中加入 $2.0 \mu \mathrm{L}$ 的気代盐酸 后, 溶液由黄色变为无色, 并伴随着黄色沉淀的生成. 重新加入気代氢氧化钠溶液后, 溶液由无色变为黄色, 同时黄色沉淀消失.



图 6 (a)主体分子 1 和 1.0 equiv. 的 2, (b) 向 a 中加入 $2.0 \mu \mathrm{L}$ 的 気代盐酸 $(20 \mathrm{wt} \%)$ 及(c)向 $\mathrm{b}$ 中加入 $1.0 \mu \mathrm{L}$ 的氛代氢氧化钠 $(40$ $\mathrm{wt} \%$ )的部分 ${ }^{1} \mathrm{H}$ NMR 谱(300 MHz, $\left.\mathrm{D}_{2} \mathrm{O}, 298 \mathrm{~K}\right)$ 和照片([1 $]_{0}=$ $4.0 \mathrm{mmol} / \mathrm{L}$ )

Figure 6 Partial ${ }^{1} \mathrm{H}$ NMR spectra $(300 \mathrm{MHz}, 298 \mathrm{~K})$ and images in $\mathrm{D}_{2} \mathrm{O}$ of (a) 1 and 1.0 equiv. of 2 , (b) after addition of $2.0 \mu \mathrm{L}$ $\mathrm{DCl}(20 \mathrm{wt} \%)$ to a, and (c) after addition of $1.0 \mu \mathrm{L} \mathrm{NaOD} \mathrm{(40}$ $\mathrm{wt} \%)$ to $\mathrm{b}\left([\mathbf{1}]_{0}=4.0 \mathrm{mmol} / \mathrm{L}\right)$

\section{2 结论}

报道了水溶性圆柱形大三环主体 $\mathbf{1}$ 与联吡啶盐的络 合性能. 大三环主体 1 可以在溶液中与固态下与联吡啶 盐形成 $1: 1$ 的超分子络合物. 此外, 主体分子 1 与客体 分子的络合与解离可以通过酸碱的加入进行可逆调控. 研究结果为进一步设计和构建具有特殊结构与功能的 水溶性超分子组装体提供了条件.

\section{3 实验部分}

\section{1 仪器与试剂}

Bruker 300 型核磁共振仪[德国布鲁克公司, 溶剂为 氛代水 $\left(\mathrm{D}_{2} \mathrm{O}\right)$ ], Rigaku R-AXIS RAPID IP 单晶 $\mathrm{X}$ 射线衍 射仪(日本)。氛代盐酸 $(20 \mathrm{wt} \%$ )、氞代氢氧化钠 (40 $\mathrm{wt} \%$ )(百灵威科技有限公司)、大环主体分子 1 (按照文献 [9]合成), 其他试剂和溶剂均为市售分析纯或化学纯.

\section{2 实验方法}

\subsection{1 络合物 $\mathbf{1 \bullet 2}$ 的晶体培养和结构测定}

将物质的量之比为 $1: 1$ 的大环主体 1 与客体分子 2 溶解在去离子水中配成适当的稀溶液置于试管中, 室温 下向其扩散不良溶剂丙酮得所需晶体. 取 $0.24 \mathrm{~mm} \times$ $0.16 \mathrm{~mm} \times 0.04 \mathrm{~mm}$ 的褐色晶体进行测试, 在 $100(2) \mathrm{K}$ 下以 $\omega / 2 \theta$ 方式扫描，在 $1.67^{\circ} \leqslant \theta \leqslant 25.0^{\circ},-18 \leqslant h \leqslant$ $18,-17 \leqslant k \leqslant 17,-25 \leqslant l \leqslant 17$ 的范围内收集到 30030 个衍射数据, 其中 8595 个为独立数据 $\left(R_{\mathrm{int}}=0.0747\right)$, 晶 体结构由直接法解出, 对全部非氢原子的坐标及各向异 性参数用 SHELXS-97 程序以最小二乘法修正 $F^{2}$ 进行 精修, 最终偏离因子 $R=0.136, w R=0.345$. 络合物 $\mathbf{1 \cdot 2}$ 在剑桥晶体结构数据库的编号为 1457398 .

\section{2 .2 主客体络合比及络合常数的测定}

主体分子 $1\left(3.0 \mathrm{mmol} \cdot \mathrm{L}^{-1}\right)$ 的浓度保持不变, 并逐 渐向其中加入客体分子(客体分子的浓度从 0.1 到 2.2 equiv.), 记录主体分子中 $\mathrm{H}_{1}$ 质子的变化, 然后以 $\mathrm{H}_{1}$ 质子 的化学位移为横坐标, 以客体分子与主体分子的初始浓 度比值为纵坐标作图，对数据进行线性拟合，两条拟合 直线交点的纵坐标即为主客体的络合比.

主客体之间的络合常数是按下面的方法求得，首先 计算出主体分子 $\mathrm{H}_{1}$ 质子的最大化学位移：以客体分子 浓度 $[\mathrm{G}]$ 的倒数为横坐标, 取其小于 1 的值, 以 $\mathrm{H}_{1}$ 质子 的化学位移差值 $\Delta$ 为纵坐标, 拟合后作直线, 直线与 $y$ 轴的交点即为 $\mathrm{H}_{1}$ 质子的最大化学位移 $\Delta_{0}$. 然后利用 Scatchard 方程, 以 $P\left(P=\Delta / \Delta_{0}\right)$ 为横坐标, $P /[\mathrm{G}]$ 的值为纵 坐标，拟合后作直线，直线的斜率即为主客体络合物的 络合常数.

致谢 单晶的解析得到了湖南省有机化学重点学科 (2016)老师们的帮助，在此对他们的帮助表示诚挚的谢 意

辅助材料(Supporting Information) 主客体络合比及 络合常数的计算谱图. 这些材料可以免费从本刊网站 (http://sioc-journal.cn/)上下载.

\section{References}

[1] Pedersen, C. J. J. Am. Chem. Soc. 1967, 89, 7017.

[2] Liu, Y.; You, C. C.; Zhang, H. Y. Supramolecular Chemistry, Nankai University Publication, Tianjin, 2001 (in Chinese). (刘育, 尤长城, 张衡益, 超分子化学, 南开大学出版社, 天津, 2001.)

[3] (a) Miyauchi, M.; Takashima, Y.; Yamaguchi, H.; Harada, A. J. Am 
Chem. Soc. 2005, 127, 2984.

(b) Wang, Y.; Ma, N.; Wang, Z.; Zhang, X. Angew. Chem., Int. Ed. 2007, 46, 2823.

(c) Xu, M.; Wu, S.; Zeng, F.; Yu, C. Langmuir 2010, 26, 4529.

(d) Chen, Y.; Liu, Y. Chin. J. Org. Chem. 2012, 32, 805 (in Chinese).

(陈涌, 刘育, 有机化学, 2012, 32, 805.)

[4] (a) Jeon, Y.-M.; Kim, J.; Whang, D.; Kim, K. J. Am. Chem. Soc. 1996, 118, 9790.

(b) Kim, Y.; Kim, H.; Ko, Y. H.; Selvapalam, N.; Rekharsky, M. V.; Inoue, Y.; Kim, K. Chem. Eur. J. 2009, 15, 6143.

(c) Liu, Y.; Yu, Y.; Gao, J.; Wang, Z.; Zhang, X. Angew. Chem., Int. Ed. 2010, 49, 6576.

(d) Jiang, W.; Wang, Q.; Linder, I.; Klautzsch, F.; Schalley, C. A. Chem. Eur. J. 2011, 17, 2344.

(e) Han, B. H.; Liu, Y. Chin. J. Org. Chem. 2003, 23, 139 (in Chinese).

(韩宝航, 刘育, 有机化学, 2003, 23, 139.)

(f) Hou, Z. S.; Tan, Y. B.; Xu, J.; Zhou, Q. F. Chin. J. Org. Chem. 2005, 25, 934 (in Chinese).

(侯昭升，谭业邦，许静，周其风，有机化学， 2005, 25, 934.)

(g) Liu, J. J.; Xu, Y.; Tian, H. Chin. J. Org. Chem. 2007, 27, 541 (in Chinese).

(刘瀷军, 许蕴, 田禾, 有机化学, 2007, 27, 541.)

[5] (a) Corbellini, F.; Knegtel, R. M.; Grootenhuis, P. D.; Crego-Calama, M.; Reinhoudt, D. N. Chem. Eur. J. 2004, 11, 298.

(b) Liu, Y.; Guo, D.-S.; Yang, E.-C.; Zhang, H.-Y.; Zhao, Y.-L. Eur. J. Org. Chem. 2005, 2005, 162.

(c) Guo, D. S.; Wang, K.; Wang, Y. X.; Liu, Y. J. Am. Chem. Soc. 2012, 134, 10244.

(d) Kulasekharan, R.; Ramamurthy, V. Org. Lett. 2011, 13, 5092.

(e) Tu, C.; Zhu, L.; Li, P.; Chen, Y.; Su, Y.; Yan, D.; Zhu, X.; Zhou,
G. Chem. Commun. 2011, 47, 6063 .

(f) Specht, A.; Bernard, P.; Goeldner, M.; Peng, L. Angew. Chem., Int. Ed. 2002, 41, 4706.

[6] (a) Li, Z.; Yang, J.; Yu, G.; He, J.; Abliz, Z.; Huang, F. Chem. Commun. 2014, 50, 2841.

(b) Si, W.; Chen, L.; Hu, X. B.; Tang, G.; Chen, Z.; Hou, J. L.; Li, Z. T. Angew. Chem., Int. Ed. 2011, 50, 12564.

(c) Yu, G.; Xue, M.; Zhang, Z.; Li, J.; Han, C.; Huang, F. J. Am. Chem. Soc. 2012, 134, 13248.

(d) Ma, Y.; Ji, X.; Xiang, F.; Chi, X.; Han, C.; He, J.; Abliz, Z.; Chen, W.; Huang, F. Chem. Commun. 2011, 47, 12340.

(e) Chen, H.; Fan, J.; Hu, X.; Ma, J.; Wang, S.; Li, J.; Yu, Y.; Jia, X.; Li, C. Chem. Sci. 2015, 6, 197.

(f) Ma, J.; Deng, H.; Ma, S.; Li, J.; Jia, X.; Li. C. Chem. Commun. 2015, 51, 6621.

[7] (a) Cram, D. J.; Cram, J. M. Science 1974, 183, 803.

(b) Wei, P.; Yan, X.; Huang, F. Chem. Soc. Rev. 2015, 44, 815.

(c) Chen, C.-F. Chem. Commun. 2011, 47, 1674.

(d) Jiang, Y.; Chen, C.-F. Eur. J. Org. Chem. 2011, 6377.

(e) Han, Y.; Meng, Z.; Ma, Y.-X.; Chen, C.-F. Acc. Chem. Res. 2014, 47, 2026.

[8] (a) Hoffart, D. J.; Tiburcio, J.; de la Torre, A.; Knight, L. K.; Loeb, S. J. Angew. Chem., Int. Ed. 2008, 47, 97.

(b) Chen, L.; Zhang, Y. M.; Liu, Y. J. Phys. Chem. B 2012, 116, 9500 .

(c) Chen, L.; Zhang, H. Y.; Liu, Y. J. Org. Chem. 2012, 77, 9766.

(d) Ji, X.; Zhang, M.; Yan, X.; Li, J.; Huang, F. Chem. Commun. 2013, 49, 1178.

[9] Zeng, F.; Chen, C.-F. Org. Biomol. Chem. 2015, 13, 1988.

[10] Connors, K. A. Binding Constants, John-Wiley and Sons, New York, 1987. 